rate (that is, a positive or negative 'light-growth reaction') was studied by illuminating with ultraviolet while rotating the culture, and afterwards following the growth-rate directly with a vertical travelling microscope. The results show clearly that exposure to $280 \mathrm{~m} \mu$, at doses which unilaterally would cause negative curvatures, temporarily promotes the growth-rate by 50-100 per cent. The negative curvature can therefore be ascribed to acceleration of growth on the near side, and a 'lens' effect such as that invoked to explain the positive curvatures ${ }^{4}$ need not be postulated. Details of measurements of this interesting growth reaction will be published elsewhere.

We gratefully acknowledge the encouragement and valued criticism of Prof. K. V. Thimann.

This research was supported in part by a grant to Prof. K. V. Thimann from the Committee on Growth acting for the American Cancer Society, Inc. GEORGE M. CURRY*

Biological Laboratories, Hans E. GRUENT

Harvard University

Cambridge, Mass.

Feb. 28.

* National Science Foundation Research Fellow.

† Fellow in Cancer Research of the American Cancer Society.

${ }^{1}$ Gruen, H., "Growth and Curvature of Phycomyces sporangiophores", thesis, Harvard University (September 1956).

${ }^{2}$ DuBuy, H. G., and Nuernbergk, E., "Phototropismus und Wachstum der Pflanzen", III, Ergebn. d. Biol., 12, 379 (1935).

${ }^{3}$ Delbrück, M., and Reichardt, W., in "Cellular Mechanisms in Differentiation and Growth", 14th Growth Symposium (Princeto University Press, Princéton, N.J., 1956).

4 Castle, E. S., J. Gen. Physiol., 17, 49 (1933).

\section{Flagellate-to-Amœba Transformation induced in Tetramitus rostratus by Nitrogen Mustard}

Tetramitus is a coprophilic protozoon which displays flagellate, amoeboid and cyst stages in its life-cycle. Previous investigators ${ }^{1.2}$ have reported that reversible transformations between the amoboid and flagellate stages in this organism could be induced by environmental changes.

In a study of the factors involved in the changes from one stage to another, two monobacterial cultures of flagellates were isolated independently. Both these cultures have resisted all attempts to transform them back into the amœboid stage over a period of three and one-half years. These attempts included changes in oxygen tension, osmotic pressure, $p \mathrm{H}$, temperature, lighting including ultra-violet, and changes in bacterial flora. Such environmental changes only led to a decreased rate of growth and multiplication up to complete cessation, with or without death of the culture as tested by washing and transplantation.

A striking change, however, took place when 48-hr. cultures of these flagellates (growing with Escherichia coli in a yeast-peptone-salt medium) were inoculated into the same medium containing one of the nitrogen mustards, methyl-bis( $\beta$-chloroethyl)amine hydrochloride $(H N 2)$. During the time of their contact with this compound, the flagellates showed neither multiplication nor transformation. With solutions containing more than $0.2 \mathrm{mgm} . / \mathrm{ml}$., inactivity and death resulted, commencing after 24-hr. exposure. Organisms that survived exposure $(0.2 \mathrm{mgm}$. $/ \mathrm{ml}$.) were washed and transferred to preconditioned media (media in which $E$. coli had been growing for $24 \mathrm{hr}$. offering a good food supply for Tetramitus). The flagellates recovered rapidly and multiplied for about three days (approximately seven generations), then transformed into the active and apparently healthy amoboid stage, which in turn could be propagated on yeast-peptone-agar slants. When transferred to broth cultures, this amœboid stage changed back into the flagellate stage, indicating that permanent injury to the transforming mechanism had not taken place.

More detailed investigation into the conditions necessary for methyl-bis( $\beta$-chloroethyl)amine hydrochloride to provoke this change in the organism showed that time of exposure to the drug was an important factor, about $48 \mathrm{hr}$. being necessary. Successful experiments were conducted with a concentration of $0.2 \mathrm{mgm} . / \mathrm{ml}$. at $p \mathrm{H} 5 \cdot 10$. As the $p H$ was shifted towards the alkaline side, the experiments failed. This might have been due, in part, to the fact that this nitrogen mustard becomes rapidly unstable at a $p H$ higher than $5 \cdot 10$, and thus may not have had sufficient time to provoke the biological change. Since it deteriorates slowly even at $p \mathrm{H} 5 \cdot 10$, it appears necessary for a sufficient concentration to be present at the start in order to maintain activity for at least $48 \mathrm{hr}$.

That nitrogen mustard acts on nucleic acid synthesis and perhaps in this way causes its effects on growth is well known; also accepted is the concept that transformations probably involve nuclear control. Perhaps components within the nucleus are capable of blocking certain cytoplasmic functions which mediate transformation. Nitrogen mustard, a known nuclear poison, could induce resistant flagellates to return to the amceboid stage (in two separate otherwise resistant cultures) by the destruction of such nuclear inhibitors. As a consequence, continued growth through seven generations after treatment may have lowered the concentration of the nuclear inhibitor(s) (not being replaced due to nuclear damage) and thereby enabled reversal to take place. In view of the above findings, it is suggested that components arise in the nucleus which render the organisms insusceptible to milder environmental changes ordinarily credited with causing transformation.

This work forms a part of a broader investigation of cyclic changes to be reported elsewhere.

Morgan M. Brent

Department of Bacteriology and Immunology, Jefferson Medical College,

Philadelphia.

Feb. 6.

1 Rafalko, J. S., J. Morph., 89, 83 (1951).

${ }^{2}$ Hollande, A., C.R. Acad. Sci., Paris, 205, 1440 (1937).

\section{Production of an Antibiotic by}

\section{Dermatophytes living in Horn Products}

THE question whether or not soil-fungi capable of producing antibiotics produce such substances in their natural habitat has been much discussed. Many exporiments have yielded contradictory results, but in these great deviation from natural conditions often occurred. However, the recent work of Wright ${ }^{1}$, on the production of gliotoxin in soil to which a few pieces of wheat straw has been added, seems to be quite convincing.

It has been shown in our laboratories that the majority of dermatophytes cultivated from fungus diseases of the human skin are capable of producing 